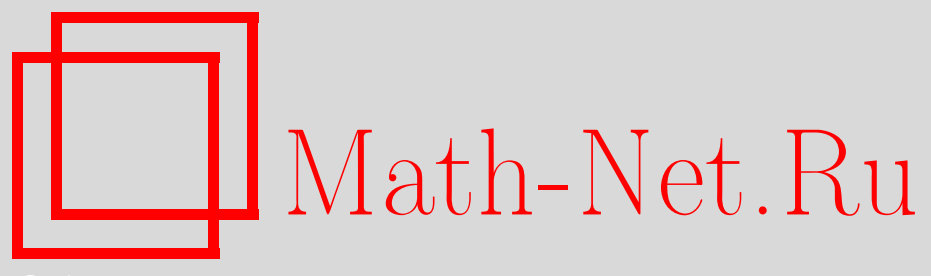

М. В. Иоффе, Ф. Канната, Д. Н. Нишнианидзе, Точно решаемая двумерная комплексная модель с вещественным спектром, ТM $\Phi, 2006$, том 148, номер 1, 102-111

DOI: https://doi.org/10.4213/tmf2061

Использование Общероссийского математического портала Math-Net.Ru подразумевает, что вы прочитали и согласны с пользовательским соглашением http://www . mathnet.ru/rus/agreement

Параметры загрузки:

IP: 35.173 .219 .149

26 апреля 2023 г., 18:37:19

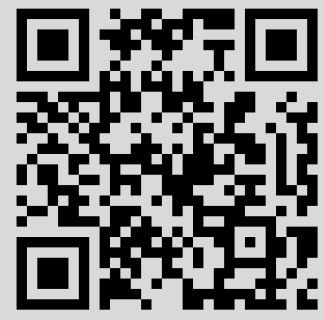




\title{
ТОЧНО РЕШАЕМАЯ ДВУМЕРНАЯ КОМПЛЕКСНАЯ МОДЕЛЬ С ВЕЩЕСТВЕННЫМ СПЕКТРОМ
}

\begin{abstract}
С помощью суперсимметричных соотношений сплетания второго порядка по производным построена двумерная квантовая модель с комплексным потенциалом, для которой все энергетические уровни и соответствующие им волновые функции найдены аналитически. Данная модель не допускает разделения переменных и может рассматриваться как один из вариантов комплексификации обобщенной двумерной модели Морса с дополнительным слагаемым $\mathrm{sh}^{-2}$. Доказана вещественность спектра этой модели. Насколько нам известно, это достаточно редкий пример нетривиальной точно решаемой двумерной модели. В явном виде получен оператор симметрии, описан биортогональный базис, и продемонстрирована псевдоэрмитовость гамильтониана модели. Полученные волновые функции одновременно являются собственными функциями оператора симметрии.
\end{abstract}

Ключевые слова: суперсимметричная квантовая механика, соотношения сплетания, комплексные потенциалы.

Статья посвящается 80-летию Юрия Викторовича Новожилова

\section{1. ВВЕДЕНИЕ}

Несмотря на то что роль точно решаемых и частично (квазиточно) решаемых моделей в одномерной квантовой механике велика ${ }^{1)}$, известно лишь сравнительно небольшое число таких моделей. Нахождение (точно и частично) решаемых систем в двумерном случае является гораздо более сложной задачей. Кроме моделей с разделением переменных, которые сводятся к паре одномерных моделей, из точно решаемых известны только модели типа Калоджеро [1] (с числом частиц $N=3)^{2}$.

1) Здесь и далее мы называем систему точно решаемой, если для нее известны аналитически энергии всех связанных состояний и соответствующие им волновые функции. Система называется частично (квазиточно) решаемой, если известна только часть из них.

* Санкт-Петербургский государственный университет, Санкт-Петербург, Россия.

${ }^{\dagger}$ Departamento de Fisica Teorica, Atomica y Optica, Universidad de Valladolid, Valladolid, Spain. E-mail: m.ioffe@pobox.spbu.ru

${ }^{\ddagger}$ Dipartimento di Fisica and INFN, Bologna, Italy. E-mail: cannata@bo.infn.it

$\S$ Технический университет Кутаиси, Кутаиси, Грузия. E-mail: qutaisi@hotmail.com 
Некоторые частично решаемые модели были построены в работах [3]-[6] с помощью суперсимметричного метода SUSY-разделения переменных. Именно методы суперсимметрии представляются наиболее подходящими для решения важной проблемы построения точно и квазиточно решаемых квантовых (и классических [7]) моделей в случае нескольких пространственных измерений.

Первый шаг в этом направлении был сделан в работах [8], где было найдено несколько частных решений суперсимметричных соотношений сплетания для двумерных систем. Все эти системы являются интегрируемыми, т.е. для них существует динамическая симметрия четвертого порядка по импульсам, и соответствующие генераторы находятся в инволюции с гамильтонианами. После этого были предложены два новых суперсимметричных метода: SUSY-разделение переменных и форминвариантность [3], [5], [6], [9], с помощью которых оказалось возможным получить часть спектра и волновых функций этих интегрируемых моделей. Таким образом, был построен класс частично решаемых интегрируемых двумерных квантовых систем. Естественно, было бы очень важно найти среди этих интегрируемых моделей какие-то точно решаемые.

В работе предлагается новый подход к проблеме, сформулированной выше. А именно, выбирая специальное значение одного из параметров $(a=-1 / 2)$ в двумерном потенциале Морса с дополнительным членом $\mathrm{sh}^{-2}$ [3], можно добиться, что один из партнеров в SUSY-соотношениях сплетания будет допускать обычное разделение переменных. В этом случае он будет точно решаемым, и его спектр и собственные функции будут найдены аналитически. При этом второй из партнеров по-прежнему не допускает разделения переменных, но, благодаря соотношениям сплетания, действие суперзаряда позволяет найти аналитически его спектр и волновые функции. Поскольку SUSY-сплетание связывает спектры с точностью до нормируемых 0-мод суперзарядов, условие точной решаемости обоих гамильтонианов требует, чтобы все 0-моды суперзарядов были известны. Все это можно сделать для обобщенной двумерной модели Морса (с дополнительным слагаемым sh${ }^{-2}$ ), но, как будет показано в разделе 2, непосредственный анализ сингулярностей волновых функций при $a=-1 / 2$ в общем виде оказывается сложным из-за присутствия в выражениях вырожденных гипергеометрических функций. Тем не менее основные возможности предложенного метода демонстрируются для некоторых энергетических уровней. В разделе 3 рассмотрение конкретного комплексного варианта этой модели (регуляризующего отталкивающие сингулярности при вещественных $\left.x_{1}, x_{2}\right)$ приводит к более интересной системе: энергии всех связанных состояний и соответствующие нормируемые волновые функции могут быть получены явно. Исследуются свойства этой модели. Главное из них - вещественность спектра (и независимость его от параметра комплексификации $\delta$ ). Кроме того, в разделе 4 стандартным образом строится биортогональный базис для связанных состояний [10] и вычисляется действие оператора симметрии на волновые функции. В частности, показано, что полученные

2) Следует упомянуть, что, как и модели Калоджеро, рассматриваемые нами модели (в том числе обобщенная модель Морса) могут использоваться [2] либо для описания трех частиц на прямой, либо одной частицы в двумерном пространстве. 
волновые функции являются общими собственными функциями как для гамильтониана, так и для оператора симметрии.

\section{2. ОБОБЩЕННЫЙ ДВУМЕРНЫЙ ПОТЕНЦИАЛ МОРСА ПРИ $a=-1 / 2$}

Основным элементом двумерной суперсимметричной квантовой механики [5], [8] второго порядка являются соотношения сплетания

$$
\widetilde{H} Q^{+}=Q^{+} H, \quad Q^{-} \widetilde{H}=H Q^{-}
$$

между двумя скалярными гамильтонианами $H, \widetilde{H}$ :

$$
H=-\triangle+V(\vec{x}), \quad \widetilde{H}=-\triangle+\widetilde{V}(\vec{x}), \quad \triangle \equiv \partial_{1}^{2}+\partial_{2}^{2}, \quad \partial_{i} \equiv \frac{\partial}{\partial x_{i}}, \quad i=1,2 .
$$

Эти соотношения позволяют установить изоспектральность суперпартнеров $H, \widetilde{H}$ с точностью до 0-мод $Q^{ \pm}$, а также связь волновых функций партнеров для одинаковых значений энергии:

$$
\Psi_{n}(x)=Q^{-} \widetilde{\Psi}_{n}(x), \quad \widetilde{\Psi}_{n}(x)=Q^{+} \Psi_{n}(x), \quad n=0,1,2, \ldots .
$$

Хотя при решении соотношений сплетания (1) нужно использовать самый общий вид суперзарядов $Q^{ \pm}$как дифференциальных операторов второго порядка по производным (подробности см. в работах [5], [8]), мы ограничимся частным решением лоренцева (гиперболического) типа:

$$
\begin{aligned}
& Q^{+}=\left(\partial_{1}^{2}-\partial_{2}^{2}\right)+C_{i} \partial_{i}+B=4 \partial_{+} \partial_{-}+C_{+} \partial_{-}+C_{-} \partial_{+}+B, \\
& Q^{-}=\left(\partial_{1}^{2}-\partial_{2}^{2}\right)-C_{i} \partial_{i}+B=4 \partial_{+} \partial_{-}-C_{+} \partial_{-}-C_{-} \partial_{+}+B, \quad i=1,2 .
\end{aligned}
$$

В этом случае потенциалы $\widetilde{V}(\vec{x}), V(\vec{x})$ и функция $B(\vec{x})$ могут быть выражены через четыре функции $F_{1}\left(2 x_{1}\right), F_{2}\left(2 x_{2}\right)$ и $C_{ \pm}\left(x_{ \pm}\right)$:

$$
\begin{aligned}
\widetilde{V} & =\frac{1}{2}\left(C_{+}^{\prime}+C_{-}^{\prime}\right)+\frac{1}{8}\left(C_{+}^{2}+C_{-}^{2}\right)+\frac{1}{4}\left(F_{2}\left(x_{+}-x_{-}\right)-F_{1}\left(x_{+}+x_{-}\right)\right), \\
V & =-\frac{1}{2}\left(C_{+}^{\prime}+C_{-}^{\prime}\right)+\frac{1}{8}\left(C_{+}^{2}+C_{-}^{2}\right)+\frac{1}{4}\left(F_{2}\left(x_{+}-x_{-}\right)-F_{1}\left(x_{+}+x_{-}\right)\right), \\
B & =\frac{1}{4}\left(C_{+} C_{-}+F_{1}\left(x_{+}+x_{-}\right)+F_{2}\left(x_{+}-x_{-}\right)\right) .
\end{aligned}
$$

Эти функции должны удовлетворять уравнению

$$
\partial_{-}\left(C_{-} F\right)=-\partial_{+}\left(C_{+} F\right),
$$

где $x_{ \pm} \equiv x_{1} \pm x_{2}, \quad \partial_{ \pm}=\partial / \partial x_{ \pm}$и $C_{ \pm}$зависят только от $x_{ \pm}$, соответственно:

$$
C_{+} \equiv C_{1}-C_{2} \equiv C_{+}\left(x_{+}\right), \quad C_{-} \equiv C_{1}+C_{2} \equiv C_{-}\left(x_{-}\right),
$$

а также

$$
F=F_{1}\left(x_{+}+x_{-}\right)+F_{2}\left(x_{+}-x_{-}\right)
$$


Среди частных решений из этого класса [5], [8] мы рассмотрим здесь хорошо изученное двумерное обобщение одномерного потенциала Морса. Эта модель (для которой в общем случае нет разделения переменных) имеет вид

$$
\begin{aligned}
& C_{+}=4 a \alpha, \quad C_{-}=4 a \alpha \operatorname{cth} \frac{\alpha x_{-}}{2}, \\
& f_{1}\left(x_{1}\right) \equiv \frac{1}{4} F_{1}\left(2 x_{1}\right)=-A\left(e^{-2 \alpha x_{1}}-2 e^{-\alpha x_{1}}\right), \\
& f_{2}\left(x_{2}\right) \equiv \frac{1}{4} F_{2}\left(2 x_{2}\right)=+A\left(e^{-2 \alpha x_{2}}-2 e^{-\alpha x_{2}}\right), \\
& \widetilde{V}(\vec{x})=\alpha^{2} a(2 a-1) \operatorname{sh}^{-2}\left(\frac{\alpha x_{-}}{2}\right)+4 a^{2} \alpha^{2}+ \\
& \quad+A\left[e^{-2 \alpha x_{1}}-2 e^{-\alpha x_{1}}+e^{-2 \alpha x_{2}}-2 e^{-\alpha x_{2}}\right], \\
& V(\vec{x})=\alpha^{2} a(2 a+1) \operatorname{sh}^{-2}\left(\frac{\alpha x_{-}}{2}\right)+4 a^{2} \alpha^{2}+ \\
& \quad+A\left[e^{-2 \alpha x_{1}}-2 e^{-\alpha x_{1}}+e^{-2 \alpha x_{2}}-2 e^{-\alpha x_{2}}\right],
\end{aligned}
$$

где параметры $a, A>0, \alpha>0$ - произвольные вещественные числа.

Именно для этой модели впервые были применены два новых метода: суперсимметричное разделение переменных и двумерная форминвариантность [3], [5]. С их помощью была продемонстрирована частичная решаемость этой модели [3], [5], [9] и аналитически получено множество волновых функций $\Psi_{n}, \widetilde{\Psi}_{n}$ для параметров, лежащих в области

$$
a \in\left(-\infty,-\frac{1}{4}-\frac{1}{4 \sqrt{2}}\right), \quad \frac{\sqrt{A}}{\alpha}-n-\frac{1}{2}>-2 a>0, \quad n=0,1,2, \ldots,
$$

что обеспечивает нормируемость 0 -мод оператора $Q^{+}$и отсутствие "падения на центр" (подробности см. в работах [3], [5]).

Напомним основную идею метода SUSY-разделения переменных. С помощью преобразования подобия суперзаряд $Q^{ \pm}$с лоренц-метрикой (4) может быть превращен в оператор, не содержащий линейных по производным членов:

$$
\begin{aligned}
q^{ \pm} & =e^{-\chi(\vec{x})} Q^{ \pm} e^{\chi(\vec{x})}=\partial_{1}^{2}-\partial_{2}^{2}+\frac{1}{4}\left(F_{1}\left(2 x_{1}\right)+F_{2}\left(2 x_{2}\right)\right), \\
\chi(\vec{x}) & \equiv-\frac{1}{4}\left(\int C_{+}\left(x_{+}\right) d x_{+}+\int C_{-}\left(x_{-}\right) d x_{-}\right),
\end{aligned}
$$

в котором теперь разделяются переменные (поэтому метод и называется SUSYразделением переменных). Если одномерные потенциалы $-F_{1}$ и $+F_{2}$ являются точно решаемыми, то нормируемые 0-моды $\Omega_{n}(\vec{x})$ для $Q^{+}$могут быть найдены аналитически. Затем в силу (1) можно построить набор волновых функций для $H$ как линейные комбинации нормируемых 0-мод, что и приводит к частичной решаемости модели. Метод форминвариантности [3] (а также наличие второй форминвариантности [9] в модели) позволяет расширить набор значений энергии и волновых функций, получаемых аналитически. 
Ниже мы сформулируем еще один метод, в котором предполагается разделение переменных в одном из гамильтонианов-партнеров (причем это не будет выполняться для другого партнера). Выберем для нашей модели значения параметров таким образом, чтобы к $H$ была применима стандартная процедура разделения переменных. Тогда наличие соотношений сплетания и знание 0-мод суперзаряда дают нам полную информацию о спектре партнера $\widetilde{H}$, для которого нет обычного разделения переменных.

Такой подходящий выбор параметров в соотношении (12) возможен - при $a=$ $-1 / 2$ гамильтониан $H$ допускает разделение переменных:

$$
H(\vec{x})=h_{1}\left(x_{1}\right)+h_{2}\left(x_{2}\right)+\alpha^{2}, \quad h_{1}\left(x_{1}\right) \equiv-\partial_{1}^{2}-f_{1}\left(x_{1}\right), \quad h_{2}\left(x_{2}\right) \equiv-\partial_{2}^{2}+f_{2}\left(x_{2}\right),
$$

а его волновые функции с энергиями

$$
E_{n, m}=\epsilon_{n}+\epsilon_{m}+\alpha^{2}
$$

(последнее слагаемое связано с наличием свободного члена $4 a^{2} \alpha^{2}$ в (12)) могут быть записаны как

$$
\Psi_{E_{n, m}}=c_{1} \eta_{n}\left(x_{1}\right) \eta_{m}\left(x_{2}\right)+c_{2} \eta_{m}\left(x_{1}\right) \eta_{n}\left(x_{2}\right), \quad c_{1}, c_{2}=\text { const },
$$

где $\epsilon_{k}$ и $\eta_{k}(x)$ являются решениями точно решаемой одномерной задачи для стандартного потенциала Морса в терминах вырожденной гипергеометрической функции (см. [11]):

$$
\begin{gathered}
\left(-\partial^{2}+A\left(e^{-2 \alpha x}-2 e^{-\alpha x}\right)\right) \eta_{k}(x)=\epsilon_{k} \eta_{k}(x), \\
\eta_{k}=e^{-\xi / 2}(\xi)^{s_{k}} \Phi\left(-k, 2 s_{k}+1 ; \xi\right), \quad \xi \equiv \frac{2 \sqrt{A}}{\alpha} e^{-\alpha x}, \\
\epsilon_{k}=-A\left[1-\frac{\alpha}{\sqrt{A}}\left(k+\frac{1}{2}\right)\right]^{2}, \quad s_{k}=\frac{\sqrt{A}}{\alpha}-k-\frac{1}{2} .
\end{gathered}
$$

Таким образом, двумерное уравнение Шредингера с гамильтонианом $H$, разумеется, точно решается и имеет двукратное вырождение уровней с $n \neq m$.

Следующим нашим шагом будет использование соотношений сплетания (1) для получения с помощью соотношений (3) волновых функций гамильтониана $\widetilde{H}$, который не допускает разделения переменных. Из явного вида $Q^{+}$становится ясно, что из-за сингулярности $Q^{+}$при $x_{-} \rightarrow 0$ необходимо детальное исследование нормируемости функций $\widetilde{\Psi}$. Хотя полный анализ этой проблемы затруднен из-за наличия в (17) гипергеометрических функций, отдельные нормируемые собственные функции $\widetilde{H}$ можно исследовать. Так, рассматривая антисимметричные $\left(c_{1}=-c_{2}\right)$ волновые функции $\Psi_{E_{n, m}}^{\mathrm{A}}$ вида (17), можно проверить ${ }^{3)}$, что при $|n-m|=1$ они представляют собой линейные комбинации 0 -мод $\Omega_{k}(\vec{x})$ оператора $Q^{+}$:

$$
\Psi_{E_{m+1, m}}^{\mathrm{A}}=-\Psi_{E_{m, m+1}}^{\mathrm{A}}=\frac{2\left(s_{m+1}+1\right)\left(2 s_{m+1}+1\right)}{2 s_{m+1}+m+2} \sum_{k=0}^{k=m} a_{m+1, k} \Omega_{k}(\vec{x}),
$$

3) Для этого необходимо использовать некоторые элементарные соотношения между вырожденными гипергеометрическими функциями (см. книгу [11], т. 1, п. 6.3), а также свойства, выведенные в работе [3], раздел 4.4 . 
и поэтому не имеют партнеров среди связанных состояний $\widetilde{H}$. Симметричные функции $\left(c_{1}=c_{2}\right)$ из $(17)$ при $n=m \pm 1$ дают $Q^{+} \Psi_{E_{m \pm 1, m}}^{\mathrm{S}}$, которые также отсутствуют среди связанных состояний $\widetilde{H}$ из-за своего ненормируемого поведения при $x_{-} \rightarrow 0$. Таким образом, в спектр $\widetilde{H}$ определенно не входят точки $E_{m \pm 1, m}$.

Из волновой функции $\Psi_{E_{0,2}}^{\mathrm{A}}$ получается симметричная функция $\widetilde{\Psi}_{E_{0,2}}^{\mathrm{S}}$, которую можно представить в следующем виде:

$$
\widetilde{\Psi}_{E_{0,2}}^{\mathrm{S}} \equiv Q^{+} \Psi_{E_{0,2}}^{\mathrm{A}}=\alpha^{2}\left(2 s_{2}+3\right)\left(\xi_{1}-\xi_{2}\right)^{2} e^{-\left(\xi_{1}+\xi_{2}\right) / 2}\left(\xi_{1} \xi_{2}\right)^{s_{2}} .
$$

Этот пример позволяет увидеть, что подходящий выбор $c_{1}, c_{2}, n, m$ в (17) может скомпенсировать сингулярность в $Q^{+}$и обеспечить нормируемость волновой функции $\widetilde{\Psi}_{E_{n, m}}$.

В принципе кроме собственных функций $\widetilde{\Psi}$ гамильтониана $\widetilde{H}$, обсуждавшихся выше, могут существовать другие нормируемые собственные функции. В этом случае из-за соотношений сплетания действие на них оператора $Q^{-}$должно давать либо нуль, либо ненормируемые функции $\Psi$. Первая из описанных возможностей исключается при анализе 0 -мод $Q^{-}(a=-1 / 2) \equiv Q^{+}(a=+1 / 2)$, принимая во внимание, что $a=+1 / 2$ лежит вне области (13). Вторая возможность тоже не реализуется, так как все нормируемые собственные функции $\widetilde{H}$ с потенциалом (11) и $a=-1 / 2$ имеют асимптотику $\widetilde{\Psi} \sim x_{-}^{2}$ при $x_{-} \rightarrow 0$. Поэтому действие $Q^{-}$не влияет на их нормируемость, и мы возвращаемся к описанному выше случаю.

\section{3. КОМПЛЕКСИФИКАЦИЯ МОДЕЛИ}

Поскольку спектр связанных состояний $\widetilde{H}$ при $a=-1 / 2$ трудно анализировать из-за сингулярностей $\widetilde{V}(\vec{x})$ и $Q^{ \pm}$при $x_{-}=0$, естественно было бы попытаться как-то от них избавиться. В одномерной квантовой механике известно, как этого добиться [12]: сдвинуть координату в комплексную область. Это означает, что теперь нам придется, вообще говоря, иметь дело с комплексными потенциалами ${ }^{4}$.

В нашем двумерном случае ${ }^{5)}$ необходимо сдвинуть $x_{-}=x_{1}-x_{2}$, что нарушает симметрию системы относительно перестановки $x_{1} \leftrightarrow x_{2}$. Простейший способ замена

$$
\vec{x} \rightarrow \vec{x}+i \vec{\delta}, \quad \vec{\delta}=(\delta, 0)
$$

(с достаточно малым $\delta$, чтобы выполнялось $\alpha \delta \in(0, \pi / 2))$, сдвигающая сингулярность с вещественной плоскости $\left(x_{1}, x_{2}\right)$. В терминах $\xi$ в формуле (19) появится фазовый множитель: $\xi \rightarrow e^{-i \alpha \delta} \xi$. Как всегда, такой мнимый сдвиг $\vec{x}$ сохраняет вещественность спектра оператора Шредингера.

При таком сдвиге формулы раздела 2 изменяются незначительно. Оператор $Q^{-}(\vec{x}+i \vec{\delta})$ в выражении (5) сохраняет свой вид, но теперь он не является эрмитово сопряженным к $Q^{+}(\vec{x}+i \vec{\delta})$, а именно $Q^{-}=\left(\left(Q^{+}\right)^{\dagger}\right)^{\star}=\left(Q^{+}\right)^{\mathrm{t}}$. Функции (8)(10) становятся комплексными, а потенциалы $(11),(12)$ (также комплексные) теперь

\footnotetext{
4) Имеется обширная литература, касающаяся одномерных неэрмитовых гамильтонианов, начало которой положила оригинальная работа Бендера и Ботчера [13] (см., например, [14]).

5) Неэрмитовы гамильтонианы в двумерной квантовой механике исследовались в работе [4].
} 
связаны соотношениями

$$
\widetilde{V}(\vec{x}+i \vec{\delta})=\widetilde{V}^{\star}(\vec{x}-i \vec{\delta})=e^{-2 i \vec{\delta} \vec{\partial}} \widetilde{V}^{\star}(\vec{x}+i \vec{\delta}) e^{2 i \vec{\delta} \vec{\partial}}
$$

Это равенство выражает свойство псевдоэрмитовости для неэрмитовых гамильтонианов, и оно гарантирует [10] в общем случае, что спектр состоит из вещественных собственных значений и пар, комплексно-сопряженных друг к другу. В частности, в нашем случае известно, что спектр $\widetilde{H}$ вещественный.

Собственные функции $\eta_{k}(x)$ одномерного уравнения Морса (17) выражаются через вырожденные гипергеометрические функции как в формуле (19), но с $\xi \rightarrow$ $e^{-i \alpha \delta} \xi$, и остаются нормируемыми. Можно проверить, что никаких дополнительных нормируемых решений (17) не появляется для комплексных $x$. В самом деле, после подстановки $\eta_{k} \equiv e^{-\xi / 2} \xi^{s} Y(\xi)$ уравнение (18) по переменной $\xi$ превращается в вырожденное гипергеометрическое уравнение [11]. Существуют разные способы представить его общее решение. Удобным является

$$
Y(\xi)=c_{1} y_{5}(\xi)+c_{2} y_{7}(\xi)
$$

где $\xi$ включает фазовый множитель, а определения линейно независимых решений $y_{5}, y_{7}$ взяты из книги [11] (см. т. 1, п. 6.7):

$$
y_{5}=\Phi(a, b ; \xi), \quad y_{7}=e^{\xi} \Psi(b-a, b ;-\xi) .
$$

Именно наличие экспоненты в $y_{7}$ позволяет доказать, что единственный способ получить нормируемые $Y$ даже при комплексных $\xi$ - это положить $c_{2}=0$. Следовательно, условия нормируемости приводят к сотношениям (19), (20), и известен весь спектр связанных состояний (16) гамильтониана $H(\vec{x}+i \vec{\delta})$, который остается вещественным после этой комплексификации.

Снова используя соотношения сплетания (1), можно получить собственные функции

$$
\widetilde{\Psi}_{E_{n, m}}(\vec{x}+i \vec{\delta})=Q^{+} \eta_{n}\left(x_{1}+i \delta\right) \eta_{m}\left(x_{2}\right) .
$$

Благодаря отсутствию сингулярности в $Q^{+}$при $x_{-} \rightarrow 0$ они будут нормируемыми.

Следует помнить, что гамильтонианы-партнеры являются изоспектральными только с точностью до 0-мод суперзарядов. Нормируемые 0 -моды $Q^{+}$для рассматриваемой модели были построены и подробно исследованы в работе [3]. Было доказано, что из них можно построить множество линейных комбинаций, которые будут являться собственными функциями гамильтониана $H$. В частности, это может быть проделано и при $a=-1 / 2$ (когда в $H$ разделяются переменные), в результате чего получаются собственные значения [3]

$$
E_{k}=2 \epsilon_{k}+2 \alpha^{2} s_{k}=-2 \alpha^{2} s_{k}\left(s_{k}-1\right)=\epsilon_{k+1}+\epsilon_{k}+\alpha^{2}, \quad s_{k}=\frac{\sqrt{A}}{\alpha}-k-\frac{1}{2} .
$$

Для произвольных $n, m$ собственные значения (16) гамильтониана $H$ являются двукратно вырожденными: в (17) присутствуют и симметричные, и антисимметричные компоненты. Но в частном случае $n=m \pm 1$ антисимметричная комбинация 
$\Psi_{E_{m \pm 1, m}}^{\mathrm{A}}$ уничтожается оператором $Q^{+}$. В отличие от эрмитовой модели из раздела 2 для симметричной функции $\Psi_{E_{m \pm 1, m}}^{\mathrm{S}}$ ее партнер $\widetilde{\Psi}_{E_{m \pm 1, m}}^{\mathrm{A}}=Q^{+} \Psi_{E_{m \pm 1, m}}^{\mathrm{S}}$ в комплексной модели не имеет сингулярности по $x_{-} \rightarrow 0$, и, значит, соответствующие уровни энергии $E_{m \pm 1, m}$ включаются в спектр $\widetilde{H}$, но вырожденными они не будут.

Возникающие, возможно, 0-моды $Q^{-}(a)=Q^{+}(-a)$ можно исследовать тем же методом, что и в работе [3]; соответствующие собственные значения $E_{k}=-2 \alpha^{2} s_{k}+$ $2 \epsilon_{k}$. Поскольку они совпадают с $E_{k, k-1}$ из общего выражения $(16), 0$-моды $Q^{-}(a)$ не дают никаких новых собственных состояний $\widetilde{H}$.

Таким образом, комплексная модель с гамильтонианом $\widetilde{H}(\vec{x}+i \delta)$ при $a=-1 / 2$ является точно решаемой и имеет вещественный спектр:

$$
E_{n, m}=\epsilon_{n}+\epsilon_{m}+\alpha^{2} .
$$

Для значений $n=m \pm 1$ уровни будут невырожденными, при всех других $n, m$ имеется двукратное вырождение. Волновые функции $\widetilde{H}$ имеют вид

$$
\widetilde{\Psi}_{E_{n, m}}=Q^{+} \Psi_{E_{n, m}},
$$

где $\Psi_{E_{n, m}}$ даются выражениями (17) с заменой $\vec{x} \rightarrow \vec{x}+i \vec{\delta}, \vec{\delta}=(\delta, 0)$.

\section{4. ИНТЕГРИРУЕМОСТЬ И БИОРТОГОНАЛЬНОСТЬ}

Известно [5], [8], что “по построению" все гамильтонианы $\widetilde{H}, H$, участвующие в соотношениях сплетания (1) с операторами $Q^{ \pm}$второго порядка по производным, находятся в инволюции с операторами четвертого порядка по производным:

$$
\widetilde{R}=Q^{+} Q^{-}, \quad R=Q^{-} Q^{+}, \quad[H, R]=0, \quad[\widetilde{H}, \widetilde{R}]=0 .
$$

Эти операторы симметрии не сводятся к функциям от гамильтонианов ${ }^{6)}$, и поэтому все такие системы, включая рассматриваемую в настоящей работе, являются интегрируемыми.

В частном случае $a=-1 / 2$ с разделением переменных в $H$, который рассматривается в данной работе, выражения для $R$ и $\widetilde{R}$ могут быть переписаны с помощью преобразования подобия (14) и явного вида одномерных гамильтонианов $h_{1}\left(x_{1}\right), h_{2}\left(x_{2}\right)$ из (15):

$$
Q^{ \pm}=e^{ \pm \chi} q^{ \pm} e^{\mp \chi}=e^{ \pm \chi}\left(h_{2}-h_{1}\right) e^{\mp \chi}
$$

Тогда оператор симметрии $R$ для гамильтониана с разделением переменных имеет вид

$$
R=\left(h_{2}-h_{1}\right)^{2}+2 \alpha^{2}\left(h_{1}+h_{2}\right)+\alpha^{4},
$$

и его волновые функции (17) являются одновременно собственными функциями $R$ с собственными значениями

$$
r_{n, m}=\left(\epsilon_{m}-\epsilon_{n}\right)^{2}+2 \alpha^{2}\left(\epsilon_{m}+\epsilon_{n}\right)+\alpha^{4} .
$$

6) За исключением случая суперзарядов с метрикой Лапласа, который здесь не рассматривается (подробнее см. в работах [8]). 
Волновые функции (24) гамильтониана $\widetilde{H}$ с неразделяющимися переменными также являются общими для $\widetilde{R}$ :

$$
\widetilde{R} \widetilde{\Psi}_{E_{n, m}}=Q^{+} Q^{-} Q^{+} \Psi_{E_{n, m}}=r_{n, m} \widetilde{\Psi}_{E_{n, m}} .
$$

Таким образом, свойство, отмеченное в работе [3] для нескольких известных волновых функций, в рассматриваемой точно решаемой модели выполняется для всех волновых функций. Хотя почти все (при $n \neq m)$ волновые функции $\widetilde{\Psi}_{E_{n, m}}$ являются двукратно вырожденными, действие оператора симметрии $\widetilde{R}$ не перемешивает их.

Факторизованные волновые функции $\Psi_{E_{n, m}}(\vec{x}+i \vec{\delta})$ для неэрмитова гамильтониана $H(\vec{x}+i \vec{\delta})$ и комплексно-сопряженные к ним функции $\Psi_{E_{n, m}}^{\star}$ образуют так называемый биортогональный базис неэрмитова гамильтониана. Соответствующие соотношения биортогональности

$$
\begin{aligned}
\left\langle\Psi_{E_{n, m}}^{\star} \mid \Psi_{E_{n^{\prime}, m^{\prime}}}\right\rangle & =\int d^{2} x \Psi_{E_{n, m}}(\vec{x}+i \vec{\delta}) \Psi_{E_{n^{\prime}, m^{\prime}}}(\vec{x}+i \vec{\delta})= \\
& =\int d x_{1} \eta_{n}\left(x_{1}+i \delta\right) \eta_{n^{\prime}}\left(x_{1}+i \delta\right) \int d x_{2} \eta_{n}\left(x_{2}\right) \eta_{n^{\prime}}\left(x_{2}\right)= \\
& =\delta_{n n^{\prime}} \delta_{m m^{\prime}}
\end{aligned}
$$

могут быть проверены либо прямым вычислением, либо сравнением интеграла вдоль линии $x_{1}+i \delta$ с аналогичным интегралом вдоль вещественной оси $x_{1}$ для случая $\delta=0$ и вещественных волновых функций $\eta_{n}$. Отсутствие для эрмитовых гамильтонианов сингулярностей в узкой полосе между этими линиями означает, что соотношения (26) следуют из обычной ортогональности волновых функций для эрмитова гамильтониана. Наоборот, если бы рассматривалось скалярное произведение, определенное с комплексным сопряжением под интегралом (26), связь с эрмитовым случаем была бы не так очевидна.

Биортогональный базис связанных состояний для неэрмитова оператора $\widetilde{H}(\vec{x}+i \vec{\delta})$ состоит из $\widetilde{\Psi}_{E_{n, m}}=Q^{+} \Psi_{E_{n, m}}$ и $\widetilde{\Psi}_{E_{n, m}}^{\star}=\left(Q^{+}\right)^{\star} \Psi_{E_{n, m}}^{\star}$. Поскольку $Q^{-}=\left(\left(Q^{+}\right)^{\dagger}\right)^{\star}$, скалярное произведение можно представить как

$$
\left\langle\widetilde{\Psi}_{E_{n, m}}^{\star} \mid \widetilde{\Psi}_{E_{n^{\prime}, m^{\prime}}}\right\rangle=\left\langle\left(Q^{+}\right)^{\star} \Psi_{E_{n, m}}^{\star} \mid Q^{+} \Psi_{E_{n^{\prime}, m^{\prime}}}\right\rangle=\left\langle\Psi_{E_{n, m}}^{\star} \mid Q^{-} Q^{+} \Psi_{E_{n^{\prime}, m^{\prime}}}\right\rangle .
$$

Так как $\Psi_{E_{n^{\prime}, m^{\prime}}}$ является также и собственной функцией оператора симметрии $R=$ $Q^{-} Q^{+}$с собственным значением $r_{n . m}$, биортогональность для $\widetilde{H}(\vec{x}+i \vec{\delta})$ следует непосредственно из (26), что приводит к диагонализации $\widetilde{H}$ на подпространстве связанных состояний.

Благодарности. Работа частично поддержана ИНФН, Университетом Болоньи (М. В. Иоффе и Д. Н. Нишнианидзе) и Министерством Образования и Науки Испании (М. В. Иоффе, грант SAB2004-0143), а также Федеральным агентством по образованию РФ (грант РНП 2.1.1.1112). Один из авторов (М. В. Иоффе) также признателен Х. Негро и Л. Нието за гостеприимство в Вальядолиде и полезные обсуждения. 


\section{Список литературы}

[1] F. Calogero, J. Math. Phys., 10 (1969), 2191; 2197; 12 (1971), 419; M. A. Olshanetsky, A. M. Perelomov, Phys. Rep., 94 (1983), 313.

[2] M. V. Ioffe, A. I. Neelov, J. Phys. A, 35 (2002), 7613.

[3] F. Cannata, M. V. Ioffe, D. N. Nishnianidze, J. Phys. A, 35 (2002), 1389.

[4] F. Cannata, M. V. Ioffe, D. N. Nishnianidze, Phys. Lett. A, 310 (2003), 344.

[5] M. V. Ioffe, J. Phys. A, 37 (2004), 10363.

[6] M. V. Ioffe, P. A. Valinevich, J. Phys. A, 38 (2005), 2497.

[7] A. A. Andrianov, M. V. Ioffe, D. N. Nishnianidze, J. Phys. A, 32 (1999), 4641.

[8] A. A. Andrianov, M. V. Ioffe, D. N. Nishnianidze, Phys. Lett. A, 201 (1995), 103; Higher order SUSY in quantum mechanics and integrability of two-dimensional hamiltonians, solv-int/9605007; А.А. Андрианов, М.В. Иоффе, Д.Н. Нишнианидзе, ТМФ, 104 (1995), 463.

[9] F. Cannata, M. V. Ioffe, D. N. Nishnianidze, Phys. Lett. A, 340 (2005), 31.

[10] A. Mostafazadeh, J. Math. Phys., 43 (2002), 205; 2814; 3944.

[11] Г. Бейтмен, А. Эрдейи, Высшие трансцендентные функиии. T. 1-3, Наука, М., 1973.

[12] V.S. Buslaev, V. Grecchi, J. Phys. A, 26 (1993), 5541; F. Cannata, M. V. Ioffe, R. Roychoudhury, P. Roy, Phys. Lett. A, 281 (2001), 305; Z. Ahmed, Phys. Lett. A, 290 (2001), 19.

[13] C. M. Bender, S. Boettcher, Phys. Rev. Lett., 80 (1998), 5243.

[14] C. M. Bender, Introduction to PT-symmetric quantum theory, quant-ph/0501052; A. Mostafazadeh, A. Batal, J. Phys. A, 37 (2004), 11645. 